\title{
Traduction nucléaire : un garde-fou contre les protéines tronquées ?
}

Chez les procaryotes, chez lesquels aucune membrane nucléaire n'isole le matériel génétique du compartiment cytoplasmique, la traduction des ARNm a lieu au cours de leur synthèse et peut même régler le processus de transcription. Chez les eucaryotes, le dogme dominant voulait que transcription et traduction soient découplées et aient lieu dans deux compartiments distincts, noyau et cytoplasme respectivement. Toutefois, certaines observations suggéraient qu'il pourrait y avoir une traduction nucléaire. D'abord, le noyau contient toute la machinerie nécessaire à la traduction (et même une proportion élevée, $20-25 \%$, des facteurs d'initiation, protéines ribosomales...) mais on ne savait pas si ces composants étaient fonctionnels ou en transit vers le cytoplasme. Un peu de traduction avait été obtenue in vitro à partir de noyaux isolés, mais on pensait qu'elle pouvait être due à des ribosomes cytoplasmiques contaminants. Enfin, on avait observé que certains ARNm mutants possédant un codon stop prématuré pouvaient être épissés de façon alternative à l'ARNm sauvage, ce qui impliquait un mécanisme nucléaire de "contrôle qualité" de la traduction des ARNm [1].

Plus généralement, les ARNm possédant un codon stop prématuré se situant en amont du dernier exon sont soumis à un mécanisme de dégradation accélérée appelé NMD (nonsense mediated mRNA decay). Celuici permet d'éliminer les transcrits non-sens qui aboutiraient à des protéines tronquées potentiellement nuisibles, même dans les cellules hétérozygotes pour la mutation. La question fondamentale que pose ce mécanisme est celle de la distinction phase ouverte de lecture d'un codon stop présent en dehors de cette phase. Il est clair que seul un processus équivalent à la traduction permet de faire une telle distinction et il semble très probable que ce soit la machinerie normale de traduction qui effectue cette analyse. Si la traduction était uniquement cytoplasmique, comment cette machinerie saurait-elle que le codon stop rencontré se situe en amont ou en aval du dernier exon alors que celui-ci a déjà été épissé dans le noyau ? Pour résoudre ce dilemme, on a formulé l'hypothèse d'une protéine nucléaire qui marquerait la position des jonctions exon-exon après l'épissage et serait exportée avec l'ARNm dans le cytoplasme. L'ARNm serait alors «validé» en entrant dans le cytoplasme par les ribosomes qui déplacerait la protéine lors de la première traduction. Seuls les ARNm qui resteraient marqués par cette protéine seraient dégradés par la voie NMD. Une protéine (Y14) marquant les jonctions exon-exon et accompagnant l'ARNm du noyau vers le cytoplasme vient effectivement d'être identifiée, mais on ne sait pas encore si elle est effectivement responsable du déclenchement du NMD [2]. En revanche, un tel mécanisme ne permet pas d'expliquer les événements d'épissage alternatif qui éliminent les exons porteurs de codons stop prématurés et aboutissent à la production de protéines anormales, comme dans le cas de certaines maladies génétiques humaines comme la gyrate distrophy ou le syndrome de Marfan [1]. Pour permettre un tel épissage alternatif, il fallait que le codon stop soit reconnu en tant que tel dans le compartiment où l'épissage a lieu: le noyau.
Le groupe de Peter Cook à Oxford (Royaume-Uni) vient effectivement de montrer l'existence d'une traduction dans le noyau de cellules de mammifères [3]. En utilisant une méthode de perméabilisation des cellules qu'ils ont élaborée et améliorée depuis de nombreuses années pour analyser la transcription, ils ont pu marquer les sites de traduction. Ceci a été réalisé en incorporant dans les protéines naissantes de la lysine marquée (avec du tritium, de la biotine ou un groupement fluorescent) après ajout aux cellules perméabilisées d'un ARNt lysine chargé avec l'acide aminé marqué dans des conditions qui ne permettent de traduire qu'une quinzaine d'acides aminés pendant l'incubation. Cela a permis de voir les protéines en cours de synthèse avant qu'elles n'aient pu quitter les ribosomes. Ils ont ainsi pu mettre en évidence la présence de sites de traduction active qui co-localisent à haute résolution (en microscopie électronique) avec plusieurs protéines de transcription et d'épissage ainsi qu'avec les sites de transcription active (les ARN naissants sont marqués avec le BrUTP). On aurait toutefois bien aimé que les auteurs nous montrent une image en microscopie optique confocale qui aurait permis d'apprécier si la majeure partie des sites nucléaires de transcription et de traduction co-localisent. De plus, les résultats montrent que la traduction nucléaire est inhibée par des inhibiteurs de transcription, ce qui suggère que ces deux processus sont couplés. Une analyse quantitative permet d'estimer qu'un seul ribosome serait impliqué dans la traduction d'une molécule d'ARN dans le noyau alors qu'il y en aurait environ 5 par ARNm dans le cytoplasme. 
Cette traduction nucléaire pourrait être impliquée dans le "contrôle qualité » des ARNm avant leur export vers le cytoplasme et contribuer ainsi à l'élimination des messagers inaptes au service de la cellule. Il reste toutefois des zones d'ombre. En effet, il semble maintenant possible qu'il y ait au moins deux mécanismes à l'œuvre dans le «contrôle qualité » des ARNm: une première vérification des ARNm dans le noyau aurait lieu, puis ceux venant d'être exportés dans le cytoplasme seraient soumis à un deuxième contrôle qui les validerait pour les faire échapper à la dégradation par la voie NMD qui semble clairement cytoplasmique, au moins chez la levure [1]. Mais si une première traduction de vérification a lieu dans le noyau, comment expliquer que cette traduction ne déplacerait pas la protéine Y14 qui va accompagner l'ARNm dans son transit vers le cytoplasme alors que l'on vient de proposer que c'est son déplacement par les ribosomes cytoplasmiques qui validerait l'ARNm?

1. Culbertson MR. RNA surveillance. Unforeseen consequences for gene expression, inherited genetic disorders and cancer. Trends Genet 1999; $15: 74-80$.
2. Kim VN, Yong J, Kataoka N, Abel L, Diem MD, Dreyfuss G. The Y14 protein communicates to the cytoplasm the position of exon-exon junctions. EMBO J 2001 ; 8: 2062-8.

3. Iborra FJ, Jackson DA, Cook PR. Coupled transcription and translation within nuclei of mammalian cells. Science 2001 ; 293 : 1139-42.

\section{Thierry Grange}

Institut Jacques-Monod, Université Paris 7, Tour 43, 2, place Jussieu, 75251 Paris Cedex 05, France.
Indigestion de noyaux. Contrairement à ceux de la grenouille, nos globules rouges adultes n'ont plus de noyaux. Ce processus d'énucléation intervient dans la moelle au stade d'érythroblaste acidophile et aboutit à la production de réticulocytes, qui passent dans la circulation et deviennent en quelques jours des globules rouges discoïdes. Le mécanisme de cette énucléation fascine les hématologistes, mais reste mal compris: il implique d'abord l'extrusion du noyau, puis sa phagocytose et sa dégradation par les phagocytes environnants. Le rôle des cellules endothéliales avait été suspecté, puisque l'érythroblaste mûr doit traverser la barrière vasculaire pour gagner la circulation. Le macrophage central de l'îlot érythroblastique médullaire est un autre candidat sérieux. Cet îlot est une structure unique où une couronne d'érythroblastes différenciés entoure un macrophage central, à la fois nourricier (puisqu'il donne le fer) et destructeur de noyaux. Il y a beaucoup de similitude entre la dégradation «physiologique » du noyau d'érythroblastes, et la dégradation des corps apopto- tiques. Celle-ci fait intervenir de nombreuses enzymes, dont la plus connue est une désoxyribonucléase (DNase) sous contrôle de la cascade des caspases, mais d'autres peuvent aussi intervenir, notamment une endonucléase acide lysosomale indépendante des caspases, la DNase II [1]. Or, chez la souris, en l'absence de cette DNase II, une anémie sévère, isolée, constamment létale, s'installe entre 12,5 et 17 jours de développement, et s'accompagne de la persistance d'érythroblastes nucléés circulants [1]. Le défaut n'est pas intrinsèque à la lignée érythroblastique, puisque si des cellules souches hématopoḯtiques de foie foetal de souris DNase $I^{-1}$ - sont greffées à des receveurs irradiés normaux (chimères hématopoḯtiques), ces derniers produisent des globules rouges DNase $\mathrm{II}^{-/}$énucléés fonctionnels. En fait, ce sont les macrophages du foie foetal qui sont touchés: leur nombre est diminué, et ils semblent avoir une indigestion de noyaux dont la dégradation est incomplète. Lors de l'installation de l'hématopoïèse hépatique, qui fait suite à l'érythropoï̀se primitive du sac vitellin (qui, elle, ne s'accom- pagne pas d'énucléation), les macrophages phagocytent d'abord normalement les noyaux d'érythroblastes mais, ne pouvant en dégrader l'ADN, ils sont vite saturés, ce qui entraîne peut-être leur destruction. On reste un peu sur sa faim quant à l'explication de l'anémie sévère de ces embryons: il n'est pas exclu que l'activation des macrophages entraîne la sécrétion de cytokines inhibitrices qui aient aussi une responsabilité dans la létalité embryonnaire. Il semble donc bien que plusieurs voies conduisent à la dégradation de l'ADN de noyaux phagocytés ; peut-être certaines sontelles spécialisées dans une dégradation "physiologique» comme celle qui intervient lors de l'énucléation en fin de différenciation érythroblastique, mais aussi dans les kératinocytes, et les cellules de la cornée, trois tissus dans lesquels l'énucléation est l'aboutissement nécessaire de la différenciation.

[1. Krieser RJ, et al. J Biol Chem 1998; 273 : 30909-14.]

[2. Kawane, et al. Science 2001; 292 : 1546-9.] 